\title{
Development of nanostructured lipid carrier for dacarbazine delivery
}

\author{
Musallam Almousallam ${ }^{1}$ Claudia $\mathrm{Moia}^{1} \cdot$ Huijun $\mathrm{Zhu}^{1}$
}

Received: 7 April 2015/ Accepted: 21 August 2015/Published online: 21 September 2015

(C) The Author(s) 2015. This article is published with open access at Springerlink.com

\begin{abstract}
Dacarbazine (Dac) is one of the most commonly used chemotherapy drugs for treating various cancers. However, its poor water solubility, short half-life in blood circulation, low response rate and high side effect limit its application. This study aimed to improve the drug solubility and prolong drug release by developing nanostructured lipid carriers (NLCs) for Dac delivery. The NLC and Dac-encapsulated NLC were synthesized with precirol ATO 5 and isopropyl myristate as lipids, tocopheryl polyethylene glycol succinate, soybean lecithin and Kolliphor P 188 as co-surfactants. The NLCs with controlled size were achieved using high shear dispersion following solidification of oil-in-water emulsion. For Dac encapsulation, the smallest NLC with $155 \pm 10 \mathrm{~nm}$ in size, $0.2 \pm 0.01$ polydispersion index and $-43.4 \pm 2 \mathrm{mV}$ zeta potential was selected. The resultant DLC-Dac possessed size, polydispersion index and zeta potential of $190 \pm 10$, $0.2 \pm 0.01$, and $-43.5 \pm 1.2$, respectively. The drug encapsulation efficiency and drug loading were $98.5 \%$ and $14 \%$, respectively. In vitro drug release study showed a biphasic pattern, with $50 \%$ released in the first $2 \mathrm{~h}$, and the remaining released sustainably for up to $30 \mathrm{~h}$. This is the first report on the development of NLC for Dac delivery, implying that NLC could be a new potential candidate as drug carrier to improve the therapeutic profile of Dac.
\end{abstract}

Keywords Nanostructured lipid carrier · Dacarbazine . High shear dispersion · Drug delivery

Huijun Zhu

h.zhu@cranfield.ac.uk

1 Institute of Environment, Health, Risks and Futures, Cranfield University, Building 42, Bedfordshire MK43 0AL, UK

\section{Introduction}

Dacarbazine (Dac), a highly lipid-soluble and light-sensitive agent chemically known as 5-(3,3-dimethyl-1-triazenyl) imidazole-4-carboxamide, is an antineoplastic drug that has been used to treat various cancers [1]. It is the only US Food and Drug Administration (FDA)-approved chemotherapeutic agent for treating wild-type melanoma, a skin cancer that accounts for the vast majority of skin cancer deaths $[2,3]$. It is the most active single agent currently used for treating metastatic melanoma [4]. Over the last decades, the death rate has been increasing faster than most other types of cancer $[5,6]$. At least a third of the patients with early-stage melanoma will develop metastases, for which the prognosis is dismal. Presently, no treatment can prolong the overall survival of the disease.

The oral adsorption of Dac is very low [7]. It is metabolized in the liver to an alkylating agent (diazomethane) that destroys cancer cells $[8,9]$, although different mechanism of Dac-toxicity was also suggested [10]. The only available formulation for clinical use is delivered through intravenous injection. After injection at $2.6-6.8 \mathrm{mg} / \mathrm{body}$ weight, the plasma concentration of the drug reached $6 \mu \mathrm{g} / \mathrm{mL}$ with half-life around $41 \mathrm{~min}$ [11]. A single-dose of $850-1000 \mathrm{mg} / \mathrm{m}$ [2] administered once every 3 weeks was referenced as standard therapy with response rate of $13-20 \%$ of patients [12-14]. The effect could only last for 3-6 months as the melanoma cells develop resistance to drug-induced apoptosis and aberrant survival pathways during progression [15]. Like other chemotherapeutical agents, Dac also causes side effect by killing normal dividing cells. There is an imperative need for new Dac formulations that could extend the drug halflife in vivo while also achieving higher tolerable dose. 
Previous studies have demonstrated that encapsulation of therapeutic agents in nanoparticles can improve their stability, bioavailability, pharmacokinetics and safety. It has been demonstrated that nanoformulation of Dac such as nanoemulsion, cubosomes and methoxy poly nanoparticles can prolong the shelf-life, enhance therapeutic efficiency and reduce side effect [16-19]. However, these formulations showed relatively low drug encapsulation efficiency and drug loading.

To this end, nanostructured lipid carrier (NLC) has emerged as a promising alternative. Being the second generation of solid lipid nanoparticles and made of a mixture of solid and liquid lipids, NLC contains highly unordered structures that increase the capacity for drug loading while significantly reducing the water content in the final formulation [20]. NLC remains solid at room and body temperature [21]. The production of NLC is of low cost with ease for scaling up [22]. Accordingly, NLCs have been investigated for drug delivery through different routes including oral, pulmonary intravenous injection, nose-tobrain, and dermal and ocular applications [23-26]. However, there has been no report on application of NLCs for Dac delivery. This study aimed to improve the drug solubility and prolong drug release by developing nanostructured lipid carriers (NLCs) for Dac delivery. NLCs were synthesized and studied for Dac encapsulation efficiency, drug loading, in vitro drug release, and stability during storage. This study suggests that the NLC developed in this study is a potential candidate for developing new formulation of Dac for improving the therapeutic profile of the drug.

\section{Materials and methods}

\section{Materials}

Dacarbazine (Dac), stearylamine (SA), D- $\alpha$-tocopheryl polyethylene glycol succinate (TPGS), isopropyl myristate (IPM), Kolliphor ${ }^{\circledR}$ P 188, acetone and ethanol ware purchased from Sigma Aldrich (Gillingham, Dorset, UK). Precirol ATO-5 and medium-chain triglycerides (MCT) were obtained from Gattefossé (Saint Priest Cédex, France). Glycerylbehenate (GB) was supplied by Fisher Scientific (Loughborough, UK). Soybean lecithin (SL) was obtained from Cuisine Innovation (Dijon, France). Double-distilled water was collected in the laboratory from Millipore-Q Gradient $\mathrm{A} 10^{\mathrm{TM}}$ ultra-pure water system (Millipore, France).

\section{Formulation and preparation of NLCs and NLC-Dac}

Three solid lipid and liquid lipid matrices were chosen to prepare NLCs (as shown in Table 1). The preparation involved oil-in-water emulsion, evaporation and solidification followed by high shear dispersion (HSD). The solid lipid, liquid lipid and emulsifiers were mixed with $12.5 \mathrm{~mL}$ of organic solvents (equal volume of acetone and ethanol) at temperature $5{ }^{\circ} \mathrm{C}$ above their melting point. The emulsion was made by adding the oil phase drop-wise to an equal volume of aqueous phase, which contained nonionic surfactant kolliphor ${ }^{\circledR} \mathrm{P} 188$ (1-3\%) and was heated at the same temperature. The mixture was stirred for $4 \mathrm{~h}$ at $400 \mathrm{rpm}$ using a magnetic stirrer, and then solidified at $0{ }^{\circ} \mathrm{C}$ overnight. Finally, the solution was subjected to HSD with a homogenizer (T25 digital Ultra-Turrax, IKA, UK) at 10,000-15,000 rpm for up to $40 \mathrm{~min}$. The same procedure was used to achieve NLCDac, where $35 \mathrm{mg}$ of Dac was added to the oil phase.

\section{Dynamic light scattering (DLS) assay}

DLS is concerned with the measurement of particles size and dispersity in a suspension. NLCs and NLC-Dac preparations were measured with a Malvern Zetasizer (Zetasizer Nanosizer S; Malvern Instruments Ltd, Worcestershire, UK) at $25^{\circ} \mathrm{C}$ under a fixed angle of $90^{\circ}$ as described previously [27]. The measurements were obtained at $633 \mathrm{~nm}$ with a 4-m He-Ne laser. To give an average value and standard deviation for the particle size and polydisperse index (PDI), three different batches for each formula were analyzed five times (run). Prior to the measurement, all the samples were diluted with doubledistilled water to a suitable scattering intensity.

\section{Zeta potential (ZP) assay}

The electro-kinetic potential for particles in colloidal system is referred to zeta potential, indicating the level of repulsion between adjacent particles in solution. The potential difference between the dispersion medium fluid and the surface of the dispersed particle is a reflection of the stability of colloidal dispersions. The samples were diluted with double-distilled water and injected into a Zetasizer (Malvern Instruments Ltd, Worcestershire, UK). Five cycles of measurements were taken to derive an average zeta potential for each sample.

\section{Transmission electron microscopy (TEM)}

The structure and morphology of the NLCs were studied by TEM. NLC preparations were diluted with double-distilled water. A droplet of each sample was applied to a copper grid coated with carbon film and air-dried. The grid was then stained with $2 \%(\mathrm{w} / \mathrm{v})$ phosphotungstic acid (PTA) solution and dried under room temperature. The particles were examined using TEM (CM20, Philips) at an operating voltage of $200 \mathrm{kV}$. 
Table 1 Components of the NLC preparations

\begin{tabular}{|c|c|c|c|c|c|c|c|c|}
\hline \multirow[t]{2}{*}{ Formulation } & \multicolumn{3}{|c|}{ Solid lipid (mg) } & \multicolumn{2}{|c|}{ Liquid lipid (mg) } & \multicolumn{2}{|c|}{ Emulsifier (mg) } & \multirow{2}{*}{$\begin{array}{l}\text { Surfactant }(\%) \\
\text { Kolliphor }^{\circledR} \text { P } 188\end{array}$} \\
\hline & SA & GB & Precirol ATO5 & IPM & MCT & SL & TPGS & \\
\hline NLC/SI & 180 & & & 60 & & 30 & 30 & $1-3$ \\
\hline NLC/GM & & 180 & & & 60 & 30 & 30 & $1-3$ \\
\hline NLC/PI & & & 180 & 60 & & 30 & 30 & $1-3$ \\
\hline
\end{tabular}

$N L C / S I$ NLC made of lipids stearylamine (SA) and isopropyl myristate, $N L C / G M$ NLC made of lipids glyceryl behenate (GB) and medium-chain triglycerides (MCT), NLC/PI NLC made of lipids precirol ATO5 and isopropyl myristate (IPM), $S L$ soybean lecithin, TPGS D- $\alpha$-tocopheryl polyethylene glycol succinate

\section{Assessment of Dac encapsulation efficiency and loading capacity}

According to previous study, Dac can be detected with UV spectrometer at the wavelength of $330 \mathrm{~nm}$ [16]. Using this method, a standard curve for Dac detection was established. Dac was dissolved in acetone $10-100 \mu \mathrm{g} / \mathrm{mL}$ and analyzed by UV spectrometry (Double Beam UV-VIS, UV-2100 Shimadzu, Japan). The standard curve had a regression equation of $y=0.2338 x-0.1896$ with $R^{2}=0.9605$. The preparation of NLC-Dac was centrifuged at $12,500 \mathrm{rpm}, 4{ }^{\circ} \mathrm{C}$ for $45 \mathrm{~min}$ (Fresco17 Microcentrifuges, Thermo scientific, UK). The free drug in the supernatant was detected using UV spectrometer and the concentration was calculated according to the regression equation for the Dac standard curve. The encapsulation efficiency (EF) and drug loading (DL) percentage were derived from the following equations [28]:

$\mathrm{EE} \%=W_{1}-W_{2} / W_{1} \times 100$
$\mathrm{DL} \%=W_{1}-W_{2} / W_{3} \times 100$,

where $W_{1}$ amount of drug added in the NLC, $W_{2}$ amount of un-entrapped drug, $W_{3}$ amount of the lipids added.

\section{In vitro drug release study}

The preparation of NLC-Dac was diluted at $10 \%$ with PBS $(\mathrm{pH}=7.4)$ and incubated at $37^{\circ} \mathrm{C}$ in a capped centrifugation tube. At intervals of $0-30 \mathrm{~h}$, the solution was centrifuged at $12,500 \mathrm{rpm}, 4{ }^{\circ} \mathrm{C}$ for $45 \mathrm{~min}$ (Fresco17 Microcentrifuges, Thermo scientific, UK). The supernatant was then taken for analysis of free drug using UV spectrometer. The concentration of Dac in the supernatant was calculated according to the regression equation of the Dac standard curve. The drug released from NLC was expressed as the percentage of total amount of drug in the solution.

\section{Powder X-ray diffraction (PXRD) assay}

PXRD was used to determine the crystalline structure in the NLC samples. The NLC and NLC-Dac preparations were freeze-dried to obtain dried product and then subjected to PXRD analysis. The samples were fast frozen under $-45{ }^{\circ} \mathrm{C}$ in a deep-freezer overnight. Next day, they were moved into an Edwards Modulyo K4 Freeze Dryer (Thermo Electron Corporation, UK). After 48 h, the NLC and NLC-Dac powders were collected and analyzed using an X-ray Diffractometer (D5005, Siemens, UK). The samples were pressed to a slit at 2,2 and $1 \mathrm{~mm}$ and scanned at $40 \mathrm{kV}, 40 \mathrm{~mA}, 0.02^{\circ} / \mathrm{sec}$ at diffraction angle of $2 \theta$ for $1 \mathrm{~h} 6$ min under $10-90{ }^{\circ} \mathrm{C}$.

\section{Statistical analysis}

All experiments were performed at least three times and one of the representative experiment data was presented in each figure. The mean values of three replicates were expressed with standard deviation (mean \pm SD). One-way ANOVA was performed using Minitab 16 software to determine significance of comparisons. Significant difference $(P$ value $\leq 0.05)$ was indicated by asterisk.

\section{Results and discussion}

\section{Optimization of NLC synthesis}

In order to achieve NLCs with desirable characteristics, the components of lipids and the synthesis parameters were optimized. A total of 24 preparations were designed to allow optimizing the lipid matrix, surfactant concentration and the HSD time and speed. As judged by PS and PDI of the NLCs, the best results were achieved with $1 \%$ of surfactant and a sheer speed of 15,000 rpm for $30 \mathrm{~min}$ for synthesis of all the NLCs. The smallest NLC of $155 \mathrm{~nm}$ was achieved with the formulation NLC/PI (Fig. 1a, bluebars), whilst the size of the formulation NLC/SI and NLC-GM was significantly larger (compare red and green bars with blue bars). Therefore, only NLC/PI was taken for further analysis. The increase of surfactant concentration from 1 to $3 \%$ was associated with a significant increase in PS (Fig. 1a, compare blue bars). The increase of HSD time to 30 min resulted in a decrease in the PS and PDI of 
NLCs, whilst when the time exceeded 30 min, a reverse effect in PS and PDI occurred (Fig. 1b, c). The same was true when the speed exceeded $15000 \mathrm{rpm}$ (data not shown).

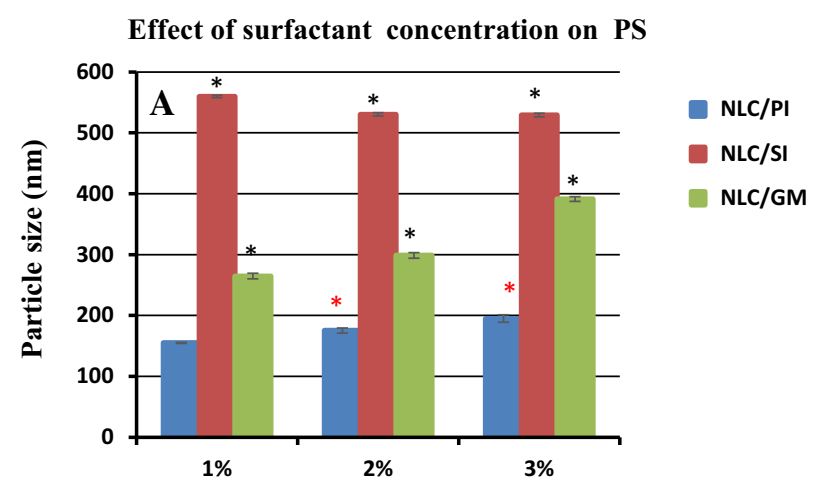

Concentration of Kolliphor P 188

Effect of HSD speed and time on PS

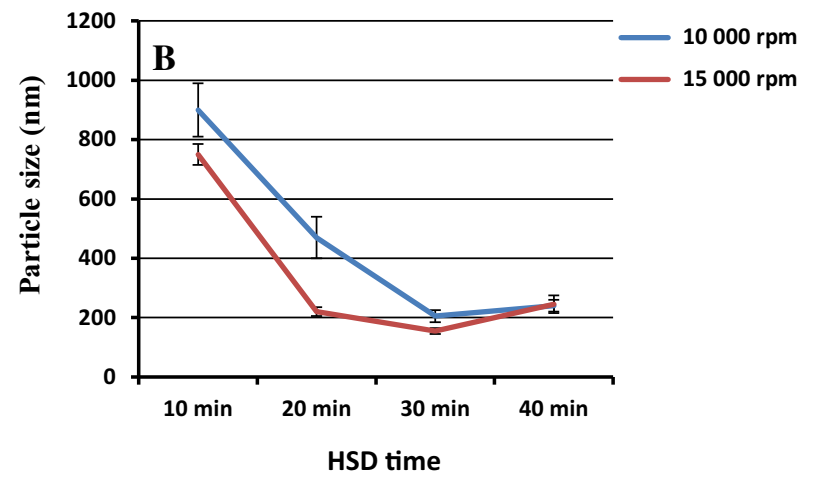

Effect of HSD speed and time on PDI

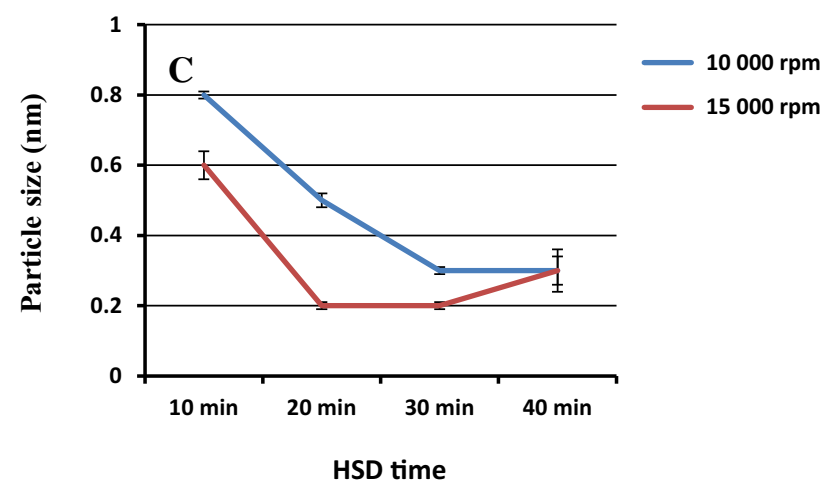

Fig. 1 Optimization of parameters used in NLC preparations. The optimal surfactant concentration and the speed and time of HSD were adjusted according to their effect on PS and PDI. a Effect of surfactant concentration on PS (black asterisks indicate significant difference as compared with blue bar at the same surfactant concentration; red asterisks indicate significant difference as compared with blue bar at $1 \%$ of surfactant. b Effect of HSD speed and time on PS of NLC/PI. c Effect of HSD speed and time on PDI of NLC/PI
Therefore, to prepare NLC/PI for further study, these optimal parameters were used.

The commonly used method for NLC synthesis involves oil-in-water emulsion, homogenization and solidification, which allows NLCs to disperse in an aqueous phase and the inner oil phase to solidify $[24,29,30]$. For different purposes modification(s) may be needed to achieve optimum preparations.

A modification has been made in this study to perform HSD after solidification. It was evident that the particle size can be controlled by the speed and time of the HSD. The failure of achieving any particles before solidification suggests that particles were formed during solidification. The application of HSD will disrupt the agglomerates of the particles, which formed possibly due to hydrophobic interaction, and also stabilize the particles by thoroughly remixing them with surfactant. The lowest particle size and PDI achieved after optimization of HSD speed and time indicated that sufficient dispersion energy was achieved and was well distributed within the solution for disruption of particle aggregates. Further increase of the HSD speed and time could result in a further reduction of PS, and consequently an increase in re-aggregation due to high inter-particle interaction force between small particles.

\section{Characteristics of NLC/PI and NLC/PI-Dac}

The NLCs were diluted with distilled water and characterized for PS, PDI and ZP. As shown in Table 2, NLC/PI exhibited a PS, PDI and ZP of $155 \pm 10 \mathrm{~nm}, 0.2 \pm 0.1$, and $-43.4 \pm 2.0$, respectively. As comparison, NLC/PI-Dac was larger with no change in PDI and little change in ZP.

Both NLC/PI and NLC/PI-Dac showed a spherical shape as observed under TEM, with NLC/PI being smaller than NLC/PI-Dac (Fig. 2, compare a with b), consistent with the results obtained by DLS measurement as shown in Table 2 . Each NLC was covered by a dark layer (Fig. 2, indicated by black arrows outside NLCs), indicative of the presence of surfactant and external solution on the surface. The internal structures of the NLC/PI and NLC/PI-Dac were very different. For NLC/PI, a large grey area (Fig. 2a, indicated by white arrow) with several white rounded core structures (Fig. 2a, indicated by black arrow inside NLC/ PI) were observed, indicative of the crystallization of solid

Table 2 Physical properties of NLC/PI and NLC/PI-Dac

\begin{tabular}{llll}
\hline Samples & PS $(\mathrm{nm})$ & PDI & ZP $(\mathrm{mV})$ \\
\hline aNLC & $155 \pm 10$ & $0.2 \pm 0.01$ & $-43.4 \pm 2.0$ \\
aNLC-Dac & $190 \pm 10$ & $0.2 \pm 0.01$ & $-43.5 \pm 1.2$ \\
\hline
\end{tabular}

Each value represents the mean $\pm \operatorname{SD}(n=5)$

$P S$ particle size, $P D I$ polydispersion index, $Z P$ zeta potential 
Fig. 2 TEM images of NLC/PI (a) and NLC/PI-Dac (b). The black arrows outside the NLCs indicate surfactant layer; the white arrows indicate liquid lipid matrix; the black arrows inside the NLCs indicate crystalised solid lipid; the dotted white arrow in $\mathbf{b}$ indicates noncrystalized solid lipid structure; the dotted black arrow in b indicates encapsulated Dac. Bar scale $50 \mathrm{~nm}$, magnification: $\times 55,000$
NLC/PI

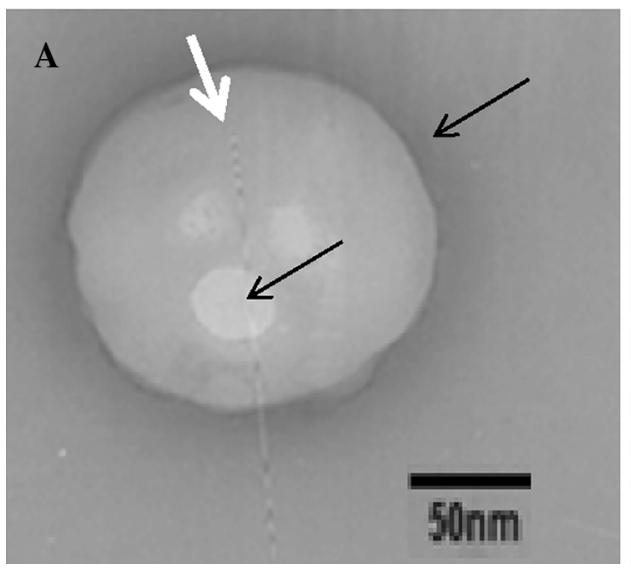

NLC/PI-Dac

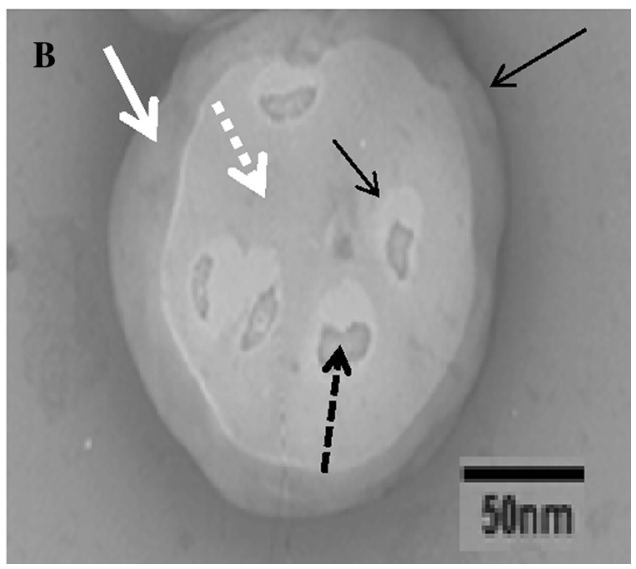

lipid within the matrix of liquid lipid. For NLC/PI-Dac, a well-defined white area (Fig. 2b, indicated by dotted white arrow) with several irregularly shaped core structures (Fig. 2b, indicated by black arrow inside NLC) was observed under a shell (Fig. 2b, indicated by white arrow). Within each core structure was a dark area (Fig. 2b, indicated by dotted black arrow), indicating the encapsulation of Dac.

The NLC/PI-Dac preparation achieved an encapsulation efficiency of $98.5 \%$ of the drug as determined using the UV detection method on day 1 after synthesis (Table 3), which was equivalent to drug loading capacity of $14 \%$. When stored in sealed amber colored glass vials in the dark at $4{ }^{\circ} \mathrm{C}$, the physical properties of the NLCs with respect to $\mathrm{PS}$, PDI and $\mathrm{EE} \%$ were stable for up to 3 months. As seen in Table 3, little change was detected over the time of storage.

$\mathrm{ZP}$ is found to be important in the colloidal stability of NPs in solution [31]. To achieve a good physical stability, $\mathrm{ZP}$ greater than $30 \mathrm{mV}$ is required, whilst for excellent stability, ZP greater than $60 \mathrm{mV}$ is required [31]. The NLC/PI and NLC/PI-Dac both presented ZP greater than $40 \mathrm{mV}$, indicating a good stability of the preparations. To achieve a good dispersion of lipid nanoparticles in aqueous phase and good stability during storage, surfactants of low toxicity and high stabilizing function have been commonly

Table 3 Stability assessment of NLC/PI-Dac storage at $4{ }^{\circ} \mathrm{C}$

\begin{tabular}{llll}
\hline Time (days) & PS & PDI & EE (\%) \\
\hline 1 & $190 \pm 10$ & $0.2 \pm 0.01$ & 98.50 \\
7 & $190 \pm 10$ & $0.2 \pm 0$ & 98.50 \\
30 & $190 \pm 10$ & $0.2 \pm 0.01$ & 95.30 \\
90 & $190 \pm 10$ & $0.2 \pm 0.02$ & 95.1 \\
\hline
\end{tabular}

$P S$ particle size, $P D I$ polydispersion index, EE \% encapsulation efficiency used [32, 33]. The surfactants selected in the current study are among the most commonly used surfactants in lipid nanoparticle preparation and appeared compatible with lipid components used in the preparation of the NLC/PI. For preparation of NLC/SI and NLC/GM, further optimizations are required to achieve desired PS and PDI.

PXRD assay was performed to characterize the crystallinity of NLC/PI and NLC/PI-Dac with Dac as a reference (Fig. 3). As reported previously, the PXRD profile of Dac presented a number of distinctive sharp peaks. Such characteristic pattern was absent in the diffraction profile of NLC/PI-Dac, indicating that Dac was no longer present as crystalline form in the NLC/PI. Some sharp distinctive peaks were also present in the PXRD profile of the NLC/PI, but their intensity was much lower in the profile of $\mathrm{NLC} /$ PI-Dac, indicating that Dac loading prevented the formation of solid lipid crystals to certain extent.

Several previous attempts have been made to develop drug delivery vehicles for Dac to improve the therapeutic profile of the drug [16, 18, 19, 34]. However, the low drug encapsulation and loading efficiency present critical limitations for these new formulations to achieve a real benefit. For example, a drug encapsulation of $70 \%$ and drug loading of $15 \mu \mathrm{g} / \mathrm{mg}(1.5 \%)$ were reported for methoxy poly (ethylene glycol)-poly (lactide) nanoparticles [18]. In comparison, the present study achieved a drug encapsulation greater than $98 \%$ and drug loading $14 \%$, which could be due to the combination of the hydrophobic nature of Precirol ATO 5 and the oily component. The high level of drug incorporation was evidenced by DLS, TEM and PXRD assays. NLC/PI-Dac was much larger than NLC/PI, indicating a substantial level of drug loading. The difference in the lipid core structures and PXRD profiles between NLC/PI and NLC/PI-Dac suggested that Dac partially prevented the crystallization of the solid lipid, leading to increased drug-lipid binding capacity. 


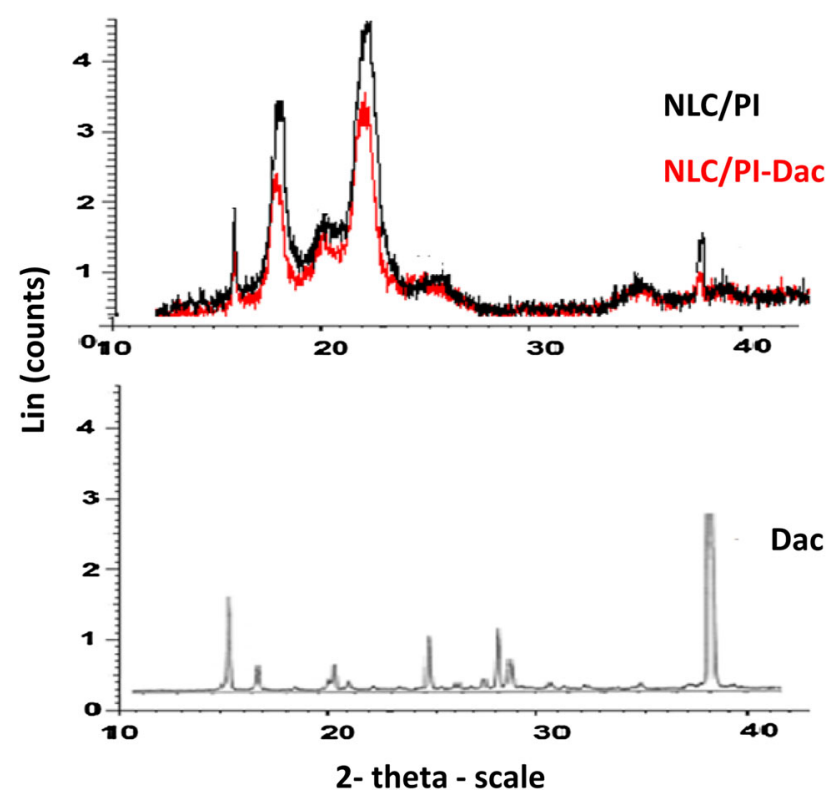

Fig. 3 PXRD pattern of Dac, NLC/PI-Dac and NLC/PI, respectively (from bottom to top). Dac exhibits a characteristic peak at $38^{\circ}$, while NLC/PI exhibits characteristic peaks at $23^{\circ}$ and $19^{\circ}$ and the intensity of the peaks reduced in NLC/PI-Dac. The $x$-axis represents the scattering angle and the $y$-axis represents the peak intensity expressed as lin counts

The release of Dac from NLC/PI in vitro appeared to follow a biphasic pattern. Nearly $50 \%$ of the drug was released from the formulation in the first $2 \mathrm{~h}$, followed by a sustainable release of the remaining drug for up to $30 \mathrm{~h}$. It has been suggested that the presence of drug on the surface and outer shell of NLC contributes to the initial burst release, whilst drug incorporated in deeper lipid phase contributes to sustained release [29]. The present study appeared to agree with this suggestion. In the first phase, Dac could be released from the outer layer of the NLC through a short diffusion path, whilst in the second phase Dac could be released in a sustained manner from the deeper liquid lipid phase and solid lipid cores through diffusion and erosion mechanisms. A similar in vitro profile for drug release from NLC containing Precirol ATO 5 was also reported previously [29, 35] (Fig. 4).

The release pattern of a drug from NLCs is determined by the nature of lipid matrix, the ability of drug to partition into both aqueous and lipid phases, temperature and surfactant concentration used to prepare the NLCs. The use of surfactant and high temperature to melt the lipid will increase the drug solubility in the aqueous phase. Upon cooling, the drug will repartition into the lipid phase and the solid lipid recrystallizes to form a solid lipid core. TPGS has been often used as an emulsifier in preparation of polymer nanoparticles [36]. It has also been recently used in combination with SL in NLC preparation, resulting

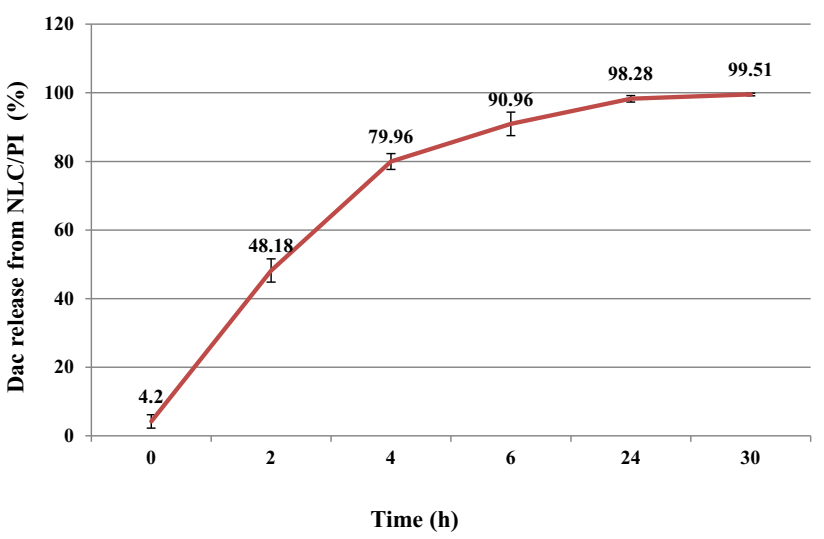

Fig. 4 In vitro drug release profile of Dac from NLC/PI in PBS $(\mathrm{pH}=7.4)$. The NLC/PI-Dac preparation was diluted at $10 \%$ in PBS and incubated at $37{ }^{\circ} \mathrm{C}$. At predetermined intervals, the release of Dac was determined by UV detection and expressed as percentage of total loaded amount of the drug

in a smaller size and higher drug EE as compared with their separate use [36, 37]. TPGS could protect the diffusion or partition of hydrophobic drug from NLC to external phase due to its big lipophilic alkyl tail (polyethylene glycol) and hydrophilic polar head portion (tocopherol succinate) [37].

\section{Potential of NLC/PI for Dac delivery}

The only formulation of Dac in clinical use is given by intravenous infusion with drug half-life less than $1 \mathrm{~h} \mathrm{[11].}$ A new formulation of Dac should prolong the drug half-life in the blood circulation, allowing enough drug to accumulate in target organ/tissue. Encapsulation of drug in NLC could provide a solution. Although NLC/PI-Dac developed in this study showed a relative earlier drug release as compared with the formulation reported by Ding et al. [15, 16], it has been demonstrated that a drug encapsulated in NLC with a similar in vitro release profile as NLC/PI-Dac developed in this study could reach the brain $2 \mathrm{~h}$ after intravenous injection [29]. For NLC/PI-Dac, although the drug in the outer layer of the NLCs could diffuse into the blood circulation, the drug incorporated inside NLC could be carried over to the side of action, therefore improving the Dac therapeutic profile. Moreover, it was suggested that lipid nanoparticles $<200 \mathrm{~nm}$ could form a monolayer on the skin and prevent the evaporation of water from the skin. By forming a transcutaneous hydration gradient, lipid nanoparticles could facilitate drug to penetrate into the deeper layers of the skin [38]. Lipidbased vehicles have been proposed for treating cutaneous melanoma and epidermoid carcinoma through topical drug delivery $[19,39]$, indicating that the NLC/PI-Dac developed could also be beneficial via topical route for which early drug release would not potentially lead to severe 
systemic toxicity. This study implicates that NLC could have the potential for drug delivery via multiple routes.

\section{Conclusion}

This is the first report on the development of NLCs for Dac delivery. The commonly used method for NLC synthesis has been modified as such that it involved oil-in-water emulsion, evaporation and solidification followed by HSD to achieve NLCs with desirable size. As compared with some existing approaches for Dac encapsulation with different materials, using NLC proved beneficial in improving the drug encapsulation and loading efficiency, prolonging drug release, storage stability, and simplified synthesis. This research suggests that NLC/PI is a new potential candidate for Dac delivery to overcome the limitations of short half-life, and low tolerant dose of the drug. Further study will be required to assess the profile of NLC/PI-Dac drug release and toxicity in vitro and in vivo.

Acknowledgments The authors acknowledge the Saudi Arabiafunded scholarship (1821) and the European commission framework 7-funded project NANODRUG (289454) for making the research possible. The authors are grateful to Kimpton Christine for technical guidance on PXRD assay and Dr. Xianwei Liu for expert support in TEM analysis at Cranfield University.

Open Access This article is distributed under the terms of the Creative Commons Attribution 4.0 International License (http://crea tivecommons.org/licenses/by/4.0/), which permits unrestricted use, distribution, and reproduction in any medium, provided you give appropriate credit to the original author(s) and the source, provide a link to the Creative Commons license, and indicate if changes were made.

\section{References}

1. Sun, M., Nie, S., Pan, X., Zhang, R., Fan, Z., Wang, S.: Quercetin-nanostructured lipid carriers: characteristics and anti-breast cancer activities in vitro. Colloid Surf. B Biointerface 113, 15-24 (2014)

2. Eigentler, T.K., Caroli, U.M., Radny, P., Garbe, C.: Palliative therapy of disseminated malignant melanoma: a systematic review of 41 randomised clinical trials. Lancet Oncol. 4(12), 748-759 (2003)

3. Jerant, A.F., Johnson, J.T., Sheridan, C.D., Caffrey, T.J.: Early detection and treatment of skin cancer. Am. Fam. Phys. 62(2), 357-368 (2000)

4. Quirin, C., Mainka, A., Hesse, A., Nettelbeck, D.M.: Combining adenoviral oncolysis with temozolomide improves cell killing of melanoma cells. Int. J. Cancer 121(12), 2801-2807 (2007)

5. Lens, M.B., Dawes, M.: Global perspectives of contemporary epidemiological trends of cutaneous malignant melanoma. Br. J. Dermatol. 150(2), 179-185 (2004)

6. Chen, J., Shao, R., Zhang, X.D., Chen, C.: Applications of nanotechnology for melanoma treatment, diagnosis, and theranostics. Int. J. Nanomedicine 8, 2677-2688 (2013)
7. Ohshio, G., Hosotani, R., Imamura, M., Sakahara, H., Ochi, J., Kubota, N.: Gastrinoma with multiple liver metastases: effectiveness of dacarbazine (DTIC) therapy. J. Hepatobiliary Pancreat. 5(3), 339-343 (1998)

8. Loo, T.L., Housholder, G.E., Gerulath, A.H., Saunders, P.H., Farquhar, D.: Mechanism of action and pharmacology studies with DTIC (NSC 45388). Cancer Treat. Rep. 60(2), 149-152 (1976)

9. Gerulath, A.H., Loo, T.L.: Mechanism of action of 5-(3,3-dimethyl-1-triazeno)imidazole-4-caboxamide in mammalian cells in culture. Biochem. Pharmacol. 21(17), 2335-2343 (1972)

10. Bedia, C., Casas, J., Andrieu-Abadie, N., Fabriàs, G., Levade, T.: Acid ceramidase expression modulates the sensitivity of A375 melanoma cells to dacarbazine. J. Biol. Chem. 286(32), 28200-28209 (2011)

11. Breithaupt, H., Dammann, A., Aigner, K.: Pharmacokinetics of dacarbazine (DTIC) and its metabolite 5-aminoimidazole-4-carboxamide (AIC) following different dose schedules. Cancer Chemother. Pharmacol. 9(2), 103-109 (1982)

12. Chapman, P.B., Einhorn, L.H., Meyers, M.L., Saxman, S., Destro, A.N., Panageas, K.S., Begg, C.B., Agarwala, S.S., Schuchter, L.M., Ernstoff, M.S., Houghton, A.N., Kirkwood, J.M.: Phase III multicenter randomized trial of the dartmouth regimen versus dacarbazine in patients with metastatic melanoma. J. Clin. Oncol. 17(9), 2745-2751 (1999)

13. Luikart, S.D., Kennealey, G.T., Kirkwood, J.M.: Randomized phase III trial of vinblastine, bleomycin, and cis-dichlorodiammine-platinum versus dacarbazine in malignant melanoma. J. Clin. Oncol. 2(3), 164-168 (1984)

14. Eggermont, A.M.M., Kirkwood, J.M.: Re-evaluating the role of dacarbazine in metastatic melanoma: what have we learned in 30 years? Eur. J. Cancer 40(12), 1825-1836 (2004)

15. Soengas, M.S., Lowe, S.W.: Apoptosis and melanoma chemoresistance. Oncogene 22(20), 3138-3151 (2003)

16. Bei, D., Zhang, T., Murowchick, J.B., Youan, B.C.: Formulation of dacarbazine-loaded cubosomes. Part III. Physicochemical characterization. AAPS PharmSciTech. 11(3), 1243-1249 (2010)

17. Ding, B., Wu, X., Fan, W., Wu, Z., Gao, J., Zhang, W., Ma, L., Xiang, W., Zhu, Q., Liu, J., Ding, X., Gao, S.: Anti-DR5 monoclonal antibody-mediated DTIC-loaded nanoparticles combining chemotherapy and immunotherapy for malignant melanoma: target formulation development and in vitro anticancer activity. Int. J. Nanomedicine 6, 1991-2005 (2011)

18. Ding, B., Zhang, W., Wu, X., Wang, X., Fan, W., Gao, S., Gao, J., Ma, L., Ding, X., Hao, Q.: Biodegradable methoxy poly (ethylene glycol)-poly (lactide) nanoparticles for controlled delivery of dacarbazine: preparation, characterization and anticancer activity evaluation. Afr. J. Pharm. Pharmacol. 5(11), 1369-1377 (2011)

19. Kakumanu, S., Tagne, J.B., Wilson, T.A., Nicolosi, R.J.: A nanoemulsion formulation of dacarbazine reduces tumor size in a xenograft mouse epidermoid carcinoma model compared to dacarbazine suspension. Nanomedicine Nanotechnol. Biol. Med. 7(3), 277-283 (2011). doi:10.1016/j.nano.2010.12.002

20. Taratula, O., Kuzmov, A., Shah, M., Garbuzenko, O.B., Minko, T.: Nanostructured lipid carriers as multifunctional nanomedicine platform for pulmonary co-delivery of anticancer drugs and siRNA. J. Control. Release 171(3), 349-357 (2013)

21. Müller, R.H., Radtke, M., Wissing, S.A.: Nanostructured lipid matrices for improved microencapsulation of drugs. Int. J. Pharm. 242(1-2), 121-128 (2002)

22. Patidar, A., Thakur, D.S., Kumar, P., Verma, J.: A review on novel lipid based nanocarriers. Int. J. Pharm. Pharm. Sci. 2(4), 30-35 (2010)

23. Alam, M.I., Baboota, S., Ahuja, A., Ali, M., Ali, J., Sahni, J.K., Bhatnagar, A.: Pharmacoscintigraphic evaluation of potential of 
lipid nanocarriers for nose-to-brain delivery of antidepressant drug. Int. J. Pharm. 470(1-2), 99-106 (2014)

24. Gonzalez-Mira, E., Egea, M.A., Souto, E.B., Calpena, A.C., García, M.L.: Optimizing flurbiprofen-loaded NLC by central composite factorial design for ocular delivery. Nanotechnology 22(4), 45101-45115 (2011)

25. Pardeike, J., Hommoss, A., Müller, R.H.: Lipid nanoparticles (SLN, NLC) in cosmetic and pharmaceutical dermal products. Int. J. Pharm. 366(1-2), 170-184 (2009)

26. Pardeike, J., Weber, S., Haber, T., Wagner, J., Zarfl, H.P., Plank, H., Zimmer, A.: Development of an Itraconazole-loaded nanostructured lipid carrier (NLC) formulation for pulmonary application. Int. J. Pharm. 419(1-2), 329-338 (2011). doi:10.1016/j. ijpharm.2011.07.040

27. Irfan, A., Cauchi, M., Edmands, W., Gooderham, N.J., Njuguna, J., Zhu, H.: Assessment of temporal dose-toxicity relationship of fumed silica nanoparticle in human lung A549 cells by conventional cytotoxicity and 1H-NMR-based extracellular metabonomic assays. Toxicol. Sci. 138(2), 354-364 (2014)

28. Neupane, Y.R., Srivastava, M., Ahmad, N., Kumar, N., Bhatnagar, A., Kohli, K.: Lipid based nanocarrier system for the potential oral delivery of decitabine: formulation design, characterization, ex vivo, and in vivo assessment. Int. J. Pharm. 477(1-2), 601-612 (2014)

29. Lim, W.M., Rajinikanth, P.S., Mallikarjun, C., Kang, Y.B.: Formulation and delivery of itraconazole to the brain using a nanolipid carrier system. Int. J. Nanomedicine 9(1), 2117-2126 (2014)

30. Puglia, C., Damiani, E., Offerta, A., Rizza, L., Tirendi, G.G., Tarico, M.S., Curreri, S., Bonina, F., Perrotta, R.E.: Evaluation of nanostructured lipid carriers (NLC) and nanoemulsions as carriers for UV-filters: characterization, in vitro penetration and photostability studies. Eur. J. Pharm. Sci. 51(1), 211-217 (2014)

31. Riddick, T.: Zeta-Meter Manual. Zeta-Meter Inc., New York (1968)
32. Kullavadee, K.O., Uracha, R., Smith, S.M.: Effect of surfactant on characteristics of solid lipid nanoparticles (SLN). Adv. Mater. Res. 364, 313-316 (2012)

33. Leonardi, A., Bucolo, C., Romano, G.L., Platania, C.B.M., Drago, F., Puglisi, G., Pignatello, R.: Influence of different surfactants on the technological properties and in vivo ocular tolerability of lipid nanoparticles. Int. J. Pharm. 470(1), 133-140 (2014)

34. Bei, D., Marszalek, J., Youan, B.C.: Formulation of dacarbazineloaded cubosomes-part I: influence of formulation variables. AAPS PharmSciTech. 10(3), 1032-1039 (2009)

35. Song, S.H., Lee, K.M., Kang, J.B., Lee, S.G., Kang, M.J., Choi, Y.W.: Improved skin delivery of voriconazole with a nanostructured lipid carrier-based hydrogel formulation. Chem. Pharm. Bull. 62(8), 793-798 (2014)

36. Mu, L., Feng, S.: Vitamin E TPGS used as emulsifier in the solvent evaporation/extraction technique for fabrication of polymeric nanospheres for controlled release of paclitaxel. J. Control. Release 80(1), 129-144 (2002)

37. Zhou, L., Chen, Y., Zhang, Z., He, J., Du, M., Wu, Q.: Preparation of tripterine nanostructured lipid carriers and their absorption in rat intestine. Die Pharmazie Int. J. Pharm. Sci. 67(4), 304-310 (2012)

38. Wissing, S.A., Müller, R.H.: Cosmetic applications for solid lipid nanoparticles (SLN). Int. J. Pharm. 254(1), 65-68 (2003). doi:10. 1016/S0378-5173(02)00684-1

39. Lei, M., Wang, J., Ma, M., Yu, M., Tan, F., Li, N.: Dual drug encapsulation in a novel nano-vesicular carrier for the treatment of cutaneous melanoma: characterization and in vitro/in vivo evaluation. RSC Adv. 5(26), 20467-20478 (2015). doi:10.1039/ C4RA16306K 